\title{
Optical constants of surface layer on gadolinium gallium garnet: ellipsometric study
}

\author{
A.I. Belyaeva, A.A. Galuza*, T.G. Grebennik, V.P. Yuriyev \\ Institute for Low Temperature Physics \& Engineering, NAS of Ukraine, 310164, Kharkov, Ukraine \\ *System Analysis \& Control Dept., Kharkov State Politechnical University, 310002, Kharkov, Ukraine
}

\begin{abstract}
A multiple angle ellipsometric method is used for measurements of thin film layers on substrates. The method evaluates fundamental optical constants and thicknesses of the film layers. Dielectric functions of the surface layers on the gadolinium gallium garnet $(\mathrm{GdGaG})$ substrate - commonly used substrate material for rare-earth ferrogarnets $(\mathrm{ReFeG})$ films, have been determined. The thickness and origin of the surface layer on the GdGaG substrate was found out. It is shown that the dielectric properties of microscopically rough layers with thicknesses $\sim$ of 20 to $35 \mathrm{~nm}$ can be accurately modeled in the homogeneous thin layer approximation, but not in the effective-medium one. The precision of data was confirmed by comparing different simulations. Agreement to the third decimal point for refraction index was shown. Errors for thicknesses were not more than 3\%.
\end{abstract}

Keywords: gadolinium gallium garnet, optical constant, surface layer, microscopic roughness, ellipsometry.

Paper received 06.10.99; revised manuscript received 16.12.99; accepted for publication 17.12.99.

\section{Introduction}

Since the development and subsequent advancement in methods used to deposit high quality epitaxial thin films of rare-earth ferrogarnets $(\mathrm{ReFeG})$, there has been a significant interest in the substrates used for these films. It is desirable that the chosen substrate provides a film that would have optimum magnetic properties. As a rule, one uses gadolinium gallium garnet ( $\mathrm{GdGaG})$ as a substrate on which epitaxial $\mathrm{ReFeG}$ can be grown. Information about crystallinity of the film, surface roughness, substrate/film interfacial roughness and the presence of reaction layers must be determined for $\mathrm{ReFeG} / \mathrm{GdGaG}$ combination. Also, how these features affect the magnetic properties must be understood. Up to now data for substrate material is not widespread. In general, the surface optical properties of $\mathrm{GdGaG}$ are different from its bulk properties. This is due to the influence of the manufacturing process, surface treatments, and aging. The manufacturing process of the $\mathrm{GdGaG}$ plate creates an optical layer on each interface between the air and GdGaG substrate. So a GdGaG plate is composed of two transparent surface layers and a bulk. These two faces are referred to as air faces. A number of phenomena, such as oxide overlayer or the unintentional modification of the substrate dielectric properties by damage from mechanical polishing, can simulate the effect of roughness, which influences significantly on the measured dielectric properties of materials, particularly as determined by ellipsometry.

In the present work, the optical constants and thickness of surface layer on $\mathrm{GdGaG}$ were determined by ellipsometry. We investigated homogeneous surface layers and effective-medium description to reveal the origin of the surface layer of microroughness on GdGaG. The method which is based on multiple angle of incidence ellipsometric data taken on a single samples at one point using a fixed wavelength $(\lambda=632.8 \mathrm{~nm})$ was applied. Ellipsometry is a nondestructive optical method that permits any small change in refractive index at the surface of a sample to be measured. This is ideal for studying our problem. Transmission electron microscope (TEM) and light scattering investigation were used to check the supposition about surface layer origin.

\section{Theory}

Ellipsometry is an optical technique that measures and interprets changes in the polarization state of light on reflection from a surface. These changes in the polarization state are characteristic of the depth profile of the dielectric function of the sample. By definition, ellipsometry measures the ratio $\rho=r_{P} / r_{S}$ of parallel $(P)$ to perpendicular $(S)$ reflectance coefficients on a sample. The electrodynamics theory applied to the ambient (a) - film (f) - substrate (s) 


\section{A.I. Belyaeva et al.: Optical constants of surface layer on gadolinium gallium garnet...}

model with constant refractive indices and sharp parallel interfaces is a classic problem in ellipso-metry. The entire optical system can be treated conventionally using the Fresnel equations. For this model the reflection factor $\rho_{c}$ at a wavelength $\lambda$ and at an angle of incidence $\theta$ is calculated [1]. The measured complex ratio is usually expressed in terms of the angles $\Psi$ and $\Delta$, where tan $\Psi$ corresponds to the relative attenuation and $\Delta$ to the phase shift between the $P$ and $S$ components of the reflectance coefficients. These angles are connected with the reflection factor by the relation [1]:

$$
\rho_{\exp }=\tan \Psi \exp (i \Delta)
$$

We equalized two reflection factors $\left(\rho_{c}, \rho_{\text {exp }}\right)$ by adjusting the unknown parameters $n_{f}$ and $d_{f}$, film refractive index and its thickness, respectively. A system of two equations (real and imaginary parts) with three parameters has to be solved. The dielectric function of a uniform, semi-infinite medium can directly be derived from Fresnel's equations. If ambient, film and substrate are transparent, one has [1]:

$$
\begin{aligned}
& \rho_{c}=\frac{R_{1 P}+R_{2 P} \exp (-2 i \delta)}{1+R_{1 P} R_{2 P} \exp (-2 i \delta)} \times \\
& \times \frac{1+R_{1 S} R_{2 S} \exp (-2 i \delta)}{R_{1 S}+R_{2 S} \exp (-2 i \delta)}
\end{aligned}
$$

where $R_{1(2) P(S)}$ - reflective Fresnel's coefficients for $P(S)$ polarizations:

$$
\begin{aligned}
& R_{1 P}=\frac{n_{f} \cos \theta-n_{a} \cos \theta_{f}}{n_{f} \cos \theta+n_{a} \cos \theta_{f}}, \\
& R_{2 P}=\frac{N_{s} \cos \theta_{f}-n_{f} \cos \theta_{s}}{N_{s} \cos \theta_{f}+n_{f} \cos \theta_{s}}, \\
& R_{1 S}=\frac{n_{a} \cos \theta-n_{f} \cos \theta_{f}}{n_{a} \cos \theta+n_{f} \cos \theta_{f}}, \\
& R_{2 S}=\frac{n_{f} \cos \theta_{f}-N_{s} \cos \theta_{s}}{n_{f} \cos \theta_{f}+N_{s} \cos \theta_{s}}, \\
& \delta=\frac{2 \pi d_{f}}{\lambda}\left(n_{f}^{2}-n_{a}^{2} \sin ^{2} \theta\right)^{1 / 2},
\end{aligned}
$$

$\delta$ is a film phase thickness; $n_{a}$ and $n_{s}$ are ambient and substrate refractive indexes, respectively. Incidence angle $\theta$ is connected with refraction angles in film $\left(\theta_{f}\right)$ and substrate $\left(\theta_{s}\right)$ by the relation: $n_{a} \sin \theta=n_{f} \sin \theta_{f}=n_{s} \sin \theta_{s}$.

Equation (2) is a quadratic in $X=\exp (-2 \mathrm{i} \delta)$ :

$C_{2} X^{2}+C_{1} X+C_{0}=0$,

where the coefficient $C_{2}, C_{1}$ and $C_{0}$ all depend nonlineary on $\Psi, \Delta, n_{a}, n_{f}, n_{s}$ and $\theta$. For transparent film the unknown $X$ satisfies the condition [1]:

$$
f_{ \pm}\left(n_{f}\right)=\left|X_{ \pm}\right|-1=0
$$

which is obviously the equation for the sole variable $n_{f}$. We have from (6):

$$
X_{ \pm}=\frac{-C_{1} \pm \sqrt{C_{1}^{2}-4 C_{0} C_{2}}}{2 C_{2}}
$$

One of the main uses of ellipsometry is determination of the refractive index $n_{f}$ and thickness $d_{f}$ of a film by the measurement of the ellipsometric ratio (1). This paper uses a method, which provides a general procedure in the analysis of ellipsometric measurements and at the same time reduces the analysis to a routine computer operation. Once the ellipsometric data were determined, a computer program was used to determine the refractive index $n_{f}$. The values of $n_{f}$ for the film was then iterated to converge the apparent thickness to a single value.

\section{Simulation}

Ellipsometry provides data $(\Psi, \Delta)$ that allow one to establish the reflection factor but not to determine directly surface physical properties. In this method the model of the sample structure must be known a priori. A mathematical representation of the sample in which the physical properties appear as parameters is used to define then.

To study the surface optical properties of one side of $\mathrm{GdGaG}$, we considered the following model: a layer with unknown refractive index $\left(n_{f}\right)$ and thickness $\left(d_{f}\right)$ overlies a well-known homogeneous substrate $\left(n_{s}\right)$. The thickness of the substrate is infinite. The system (film plus substrate) is placed in an isotropic homogeneous ambient medium (air) with a known refractive index $n_{a}$. The ambient medium, film and substrate are assumed to be transparent $\left(k_{a}=\right.$ $k_{f}=k_{s}=0$ ). We examine the case, when $n_{f}$ is constant in the thickness of the layer.

The film was modeled as a layer of surface roughness on top of an infinite plate of bulk substrate $\mathrm{GdGaG}$ material. Two models for substrate layer roughness were examined:

1) homogeneous manufactured layer and

2) a mixture of air and $\mathrm{GdGaG}$ substrate material using the Bruggeman effective medium approximation (BEMA) [2] (Fig. 1). BEMA is a natural first approximation to model a rough surface layer, that represents a heterogeneous dielectric mixture by a single parameter. This approximation leads to the following relation between $n_{s}$ and $n_{f}[2]$ :

$v_{a} \frac{1-n_{f}}{1+2 n_{f}}+\left(1-v_{a}\right) \frac{n_{s}-n_{f}}{n_{s}+2 n_{f}}=0$,

where $v_{a}$ and $\left(1-v_{a}\right)=v_{s}-$ volume fractions of air and $\mathrm{GdGaG}$ in the effective medium, respectively. In carrying out the data analysis, layer thickness and volume fraction are chosen as the fitting parameters to be determined. As we know from the literature [3], the volume fractions in the surface layer were fixed to $50 \%$ in the model if the layer thickness on the polished substrate is low. 


\section{A.I. Belyaeva et al.: Optical constants of surface layer on gadolinium gallium garnet...}

\begin{tabular}{lc}
\hline Air & $n_{a}=1$ \\
\hline Film: GdGaG($\left(1-v_{a}\right)+\operatorname{air}\left(v_{a}\right)$ \\
\hline$\left(n_{f}, d_{f}\right)$ \\
Bulk substrate GdGaG $n_{s}=1.98$ \\
\hline
\end{tabular}

Fig. 1. Schematic model for GdGaG sample. The film - substrate roughness was modeled as a mixture of air and substrate material using the Bruggeman effective medium approximation [2].

\section{Experiment}

We carried out the investigation of three GdGaG samples with different mechanical stresses in their surface layers. Samples 1, 2 and 3 passed mechanical mild abrasion test as specified for standard ferrogarnet film substrates at different departments. The samples had different damage from mechanical polishing and so various degree of roughness.

The ellipsometric measurements were fulfilled at different angles of incidence with cryogenic spectrometric ellipsometer (CSE), described in detail elsewhere [4,5], which works at multiangles of incidence and at multi-wavelengths. In our case we operated the He-Ne laser $(\lambda=632.8 \mathrm{~nm})$ as a light source at room temperature. The angles of incidence were varied from $50^{\circ}$ to $70^{\circ}$. We have used the incidence angles which were found near the principal angle of incidence $(\cos \Delta=0)$ for the calculations of the optical constants and the thickness of the sample. Four-zone averaging was used to reduce errors to about $0.05^{\circ}$ for $\Psi$ and $0.10^{\circ}$ for $\Delta$. Table 1 illustrates the typical calculated values $(\Psi, \Delta)$ for the bare substrate $\mathrm{GdGaG}\left(n_{s}=1.98[6], d_{f}=0, \lambda=632.8 \mathrm{~nm}\right)$, if we use five angles of incidence. The value $\Delta$ is defined as the difference between the phase shifts of the parallel and perpendicular components of the light. For light reflected from a transparent material $\Delta$ will be near $0^{\circ}$ or $180^{\circ}$ (Table 1 ). These values change at Brewsters angle, which for $\mathrm{GdGaG}$ is $\theta_{B}=63.17^{\circ}\left(n_{s}=\operatorname{tg} 63.17^{\circ}=1.98\right.$ at $\left.\lambda=632.8 \mathrm{~nm}\right)$.

The experimental data are illustrated by the first columns in Tables 2-4 for samples 1, 2 and 3, respectively. The refractive index $n_{\text {fexp }}$ and thickness $d_{f \exp }$ for the layers covering our GdGaG samples, which are in the tables too, have been calculated on the experimental $Y(q)$ and $D(q)$ dependencies by solving the inverse ellipsometric problem. Five experimental data points for different $q$ have been used. The aver-

Table 1. Calculated $\Psi$ and $\Delta$ values for bare substrate GdGaG $\left(n_{s}=1.98, d_{f}=0, \lambda=632.8 \mathrm{~nm}\right)$ and various incidence angles $\theta$.

\begin{tabular}{lll}
\hline \hline$\theta^{\mathrm{o}}$ & $\Delta_{c}{ }^{\mathrm{o}}$ & $\Psi_{c}{ }^{\mathrm{o}}$ \\
\hline 70 & 0 & 10.979079 \\
65 & 0 & 2.831309 \\
60 & 179.999997 & 4.888182 \\
55 & 179.999997 & 12.016903 \\
50 & 179.999987 & 18.434144 \\
\hline \hline
\end{tabular}

age values of the five incident angles for $n_{f \exp }$ (av) and $d_{f \exp }$ (av) were clearly defined, and these results are tabulated in Tables 2-4.

The accuracy of the optical constant data obtained by our method was checked by calculating data: the experimental data were directly inverted to obtain the $n_{f c}$ and $d_{f c}$. We assumed that our system has optical constants of $n_{\text {fexp }}$ (av) and $d_{\text {fexp }}$ (av). Using these parameters, we computed the five sets of $(\Psi, \Delta)$ values and treated these values as input data to the computer program after they have been rounded off to three decimal places. The numerical results are shown in the second columns of Tables 2-4. In this case the substrate roughness was modeled by homogeneous manufactured layer. We have the values of the numerical solution exactly the same as the original assumed values of the optical constants of our system. Tables 2-4 show the excellent agreement between the experiment and calculated data. The $n_{f}$ values agree throughout this range to \pm 0.001 . The difference between the two data is of the order of the instrument accuracy $\left( \pm 0.05^{\circ}\right.$ for $\Psi$ and $\pm 0.10^{\circ}$ for $\Delta$ ).

Table 5 shows the experimental data for the sample 1 . But in this case the substrate roughness was modeled as a mixture of air and substrate material GdGaG using the BEMA (Fig. 1). The volume fractions in the surface layer were fixed to $50 \%\left(v_{a}=v_{s}=0.5\right)$ in the model [3]. Fig. 2 shows the dependence of $n_{f}$ as a function of $v_{a}$, according to Eq. (8). It may be approximated by parabola $\left(n_{f}=\right.$ $\left.0.219943 v_{a}^{2}-1.20488 v_{a}+1.98248\right)$ with very good accuracy. In this approximation $v_{a}=0.11$ corresponds to $n_{f}=1.869699$, which was obtained when the substrate roughness was modeled by homogeneous manufactured layer $\left(n_{\text {fexp }}(\mathrm{av})\right.$, Table 2).

\section{Error analysis}

The influence of the real experimental errors on the numerical results for ideal air-film-GdGaG substrate system $\left(n_{s}=1.98, n_{f}=1.869699, d_{f}=33.18454, \theta=65^{\circ}, \lambda=632.8 \mathrm{~nm}\right)$ is illustrated by Table 6 . Our experimental errors for $\Psi$ and $\Delta$ are $\pm 0.05^{\circ}$ and $\pm 0.10^{\circ}$, respectively. It is quite obvious, that for $n_{f}$ we have an accuracy to the third decimal point. Errors for $d_{f}$ are not more than $3 \%$.

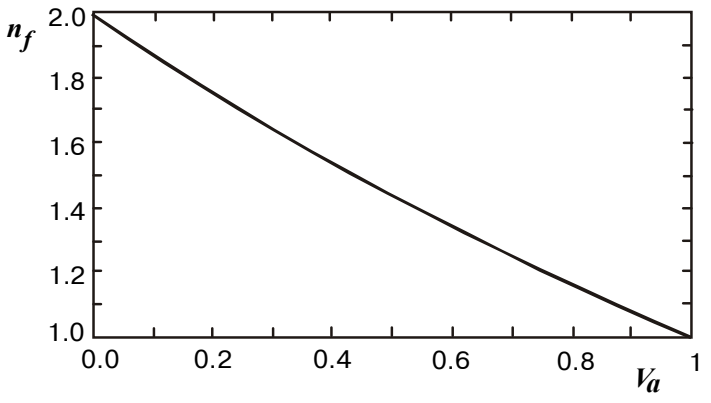

Fig. 2. The variation of film refractive index $n_{f}$ as afunction of air volume fraction $v_{a}$ using BEMA (equation (8)), $n_{s}=1.98$ 
Table 2. Experimental and numerical results for air-film-GdGaG substrate system $\left(n_{s}=1.98, \lambda=632.8 \mathrm{~nm}\right)$ for various incidence angles $\theta$.

Sample 1. The substrate roughness was modeled by homogeneous manufactured layer

\begin{tabular}{|c|c|c|c|c|c|c|c|c|}
\hline \multirow{2}{*}{$\begin{array}{l}\text { Incidence } \\
\text { angle } \\
\theta^{\mathrm{o}}\end{array}$} & \multicolumn{4}{|c|}{ Experimental data } & \multicolumn{4}{|c|}{$\begin{aligned} & \text { Numerical solution } \\
\text { Input data: } n_{f \exp }(\mathrm{av})= & 1.869699 ; d_{f \exp }(\mathrm{av})=33.18454 \mathrm{~nm}\end{aligned}$} \\
\hline & $\Delta_{\text {exp }}{ }^{o}$ & $\Psi_{\exp }^{o}$ & $n_{\text {fexp }}$ & $d_{f \mathrm{exp}}, \mathrm{nm}$ & $\Delta_{\mathrm{c}}^{\mathrm{o}}$ & $\Psi_{\mathrm{c}}{ }^{\mathrm{o}}$ & $n_{f \mathrm{c}}$ & $d_{f \mathrm{c}}, \mathrm{nm}$ \\
\hline 70 & 8.725 & 12.096 & 1.865967 & 33.244693 & 8.458987 & 12.05808 & 1.869699 & 33.184546 \\
\hline 65 & 25.792 & 4.237 & 1.868164 & 32.432982 & 25.638717 & 4.267379 & 1.869699 & 33.184547 \\
\hline 60 & 155.958 & 4.283 & 1.874264 & 33.269934 & 154.92027 & 4.286694 & 1.869699 & 33.184532 \\
\hline 55 & 170.800 & 11.237 & 1.860974 & 32.006061 & 171.250013 & 11.216039 & 1.869699 & 33.184541 \\
\hline 50 & 175.292 & 17.658 & 1.879128 & 34.969557 & 175.017164 & 17.686222 & 1.869699 & 33.184537 \\
\hline Average & & & 1.869699 & 33.18454 & & & 1.869699 & 33.184541 \\
\hline
\end{tabular}

Table 3. Experimental and numerical results for air-film-GdGaG substrate system $\left(n_{s}=1.98, \lambda=632.8 \mathrm{~nm}\right)$ for various incidence angles $\theta$.

Sample 2. The substrate roughness was modeled by homogeneous manufactured layer.

\begin{tabular}{|c|c|c|c|c|c|c|c|c|}
\hline \multirow{2}{*}{$\begin{array}{l}\text { Incidence } \\
\text { angle } \\
\theta^{\mathrm{o}}\end{array}$} & \multicolumn{4}{|c|}{ Experimental data } & \multicolumn{4}{|c|}{$\begin{array}{c}\text { Numerical solution } \\
\text { Input data: } n_{f \exp }(\mathrm{av})=1.804632 ; d_{f \exp }(\mathrm{av})=21.032552 \mathrm{~nm}\end{array}$} \\
\hline & $\Delta_{\text {exp }}{ }^{o}$ & $\Psi_{\exp }{ }^{\circ}$ & $n_{\text {fexp }}$ & $d_{f \mathrm{exp}}, \mathrm{nm}$ & $\Delta_{\mathrm{c}}^{\mathrm{o}}$ & $\Psi_{\mathrm{c}}{ }^{\mathrm{o}}$ & $n_{f \mathrm{c}}$ & $d_{f \mathrm{c}}, \mathrm{nm}$ \\
\hline 70 & 9.675 & 11.661 & 1.788521 & 19.812056 & 9.395312 & 11.689346 & 1.805904 & 21.132796 \\
\hline 65 & 29.300 & 3.967 & 1.819574 & 22.39813 & 30.138723 & 3.955846 & 1.805904 & 21.132794 \\
\hline 60 & 155.975 & 4.721 & 1.809617 & 21.188192 & 155.582765 & 4.730296 & 1.805904 & 21.1328 \\
\hline 50 & 175.325 & 17.321 & 1.800817 & 20.731803 & 174.72000 & 17.24300 & 1.805904 & 21.1328 \\
\hline Average & & & 1.804632 & 21.032552 & & & 1.805904 & 21.1328 \\
\hline
\end{tabular}

Table 4. Experimental and numerical results for air-film-GdGaG substrate system $\left(n_{s}=1.98, \lambda=632.8 \mathrm{~nm}\right)$ for various incidence angles $\boldsymbol{\theta}$.

Sample 3. The substrate roughness was modeled by homogeneous manufactured layer.

\begin{tabular}{l|l|l|l|l|l|l|l|l}
\hline \hline $\begin{array}{l}\text { Incidence } \\
\text { angle }\end{array}$ & \multicolumn{5}{|c|}{ Experimental data } & \multicolumn{5}{c}{$\begin{array}{c}\text { Numerical solution } \\
\text { Input data: } n_{f \exp }(\mathrm{av})=1.852982 ; d_{f \exp }(\mathrm{av})=25.377735 \mathrm{~nm}\end{array}$} \\
\hline$\theta^{\mathrm{o}}$ & $\Delta_{\exp }{ }^{\mathrm{o}}$ & $\Psi_{\exp }{ }^{\mathrm{o}}$ & $n_{\text {fexp }}$ & $d_{f \exp }, \mathrm{nm}$ & $\Delta_{\mathrm{c}}{ }^{\mathrm{o}}$ & $\Psi_{\mathrm{c}}{ }^{\mathrm{o}}$ & $n_{f \mathrm{c}}$ & $d_{f \mathrm{c}}, \mathrm{nm}$ \\
\hline 70 & 8.29 & 11.725 & 1.848355 & 24.775864 & 8.116622 & 11.7333 & 1.854328 & 25.502432 \\
65 & 26.3166 & 3.9875 & 1.856502 & 26.866336 & 26.143203 & 3.912335 & 1.854328 & 25.502443 \\
60 & 155.9583 & 4.2833 & 1.847600 & 24.8789. & 154.92027 & 4.286694 & 1.85433 & 25.502440 \\
55 & 172.1202 & 11.5375 & 1.851157 & 24.556131 & 172.039143 & 11.506028 & 1.854328 & 25.502446 \\
50 & 175.6167 & 17.954 & 1.861298 & 25.811442 & 175.411432 & 17.948541 & 1.854328 & 25.502428 \\
Average & & & 1.852982 & 25.377735. & & & 1.85433 & 25.50243 \\
\hline \hline
\end{tabular}

\section{Discussion}

The analysis of the experimental data shows, first of all, that our samples are not bare surface but contain the surface layers. Really, the experimental ellipsometric values $\Psi_{\exp }$ and $\Delta_{\text {exp }}$, measured on both faces of GdGaG plate (Table 2-4), are close to the ones of its bulk (Table 1). This indicates that the values of $n_{s}$ and $n_{f}$ (and Brewsters angles) are near each other. But the minor differences between experimental (Table 2-4) and calculated data (Table 1) mean that GdGaG is not bare surface and confirmed the presence of the surface layers on our samples. The thickness of the layers was determined to be $33.2 \pm 0.8 \mathrm{~nm}$ (sample 1), $21.0 \pm 0.8 \mathrm{~nm}$ (sample 2) and $25.0 \pm 0.4 \mathrm{~nm}$ (sample 3 ) (Tables 2-4). It is real values for our samples. We checked them by other methods. The samples were checked for macroscopic roughness by light scattering. The absence of macroscopic scattering shows that macroscopic roughness is not detected by usual light scattering techniques. So the assumption of negligible macroscopic roughness seems to be satisfied very well for these 


\section{A.I. Belyaeva et al.: Optical constants of surface layer on gadolinium gallium garnet...}

Table 5. Experimental and numerical results for air-film-GdGaG substrate system $\left(n_{\mathrm{s}}=1.98, \lambda=632.8 \mathrm{~nm}\right)$ for various incidence angles $q$.

Sample 1. The substrate roughness was modeled as a mixture of air and substrate material GdGaG using the Bruggeman effective medium approximation [2](Fig. 1). The volume fractions in the surface layer were fixed to $50 \%$.

\begin{tabular}{|c|c|c|c|c|c|c|c|c|}
\hline \multirow{2}{*}{$\begin{array}{l}\text { Incidence } \\
\text { angle }\end{array}$} & \multicolumn{4}{|c|}{ Experimental data } & \multicolumn{4}{|c|}{$\begin{aligned} & \text { Numerical solution } \\
& \text { Input data: } n_{f \exp }(\mathrm{av})=1.434929 ; d_{f \exp }(\mathrm{av})=9.380000 \mathrm{~nm}\end{aligned}$} \\
\hline & $\Delta_{\exp }{ }^{0}$ & $\Psi_{\exp }{ }^{0}$ & $n_{\text {fexp }}$ & $d_{\text {fexp }}, \mathrm{nm}$ & $\Delta_{\mathrm{c}}{ }^{\mathrm{o}}$ & $\Psi_{\mathrm{c}}{ }^{\mathrm{o}}$ & $n_{f \mathrm{c}}$ & $d_{f \mathrm{c}}, \mathrm{nm}$ \\
\hline 70 & 8 & 8 & 1 & 9 & 9.565 & 11. & 14 & 9.37 \\
\hline & & & & & & & & \\
\hline 60 & 1 & 4.28 & 1. & & & & & 3 \\
\hline 55 & 170.8000 & 11.2375 & 1.4349 & 9.862771 & 171 & 12.062491 & 1.43 & 9.380005 \\
\hline 50 & 175.2917 & 17.6583 & 1.434929 & 8.836721 & 174.980683 & 18.4308 & 1.434923 & 9.380003 \\
\hline Average & & & 1.434929 & 9.380000 & & & 1.434929 & 9.38 \\
\hline
\end{tabular}

Table 6. The influence of the real experimental errors on the numerical results for air-film-GdGaG substrate system $\left(n_{s}=1.98, \theta=65^{\circ}, \lambda=632.8 \mathrm{~nm}\right)$.

\begin{tabular}{l|l|l|l}
\hline \hline$\Delta_{\text {exp }}{ }^{\mathrm{o}}$ & $\Psi_{\exp }{ }^{\mathrm{o}}$ & $n_{f}$ & $d_{f}, \mathrm{~nm}$ \\
\hline $25.792+0.00$ & $4.237+0.00$ & 1.8682 & 32.4330 \\
$25.792+0.10$ & $4.237+0.05$ & 1.8681 & 33.1463 \\
$25.792-0.10$ & $4.237-0.05$ & 1.8681 & 31.6889 \\
$25.792-0.10$ & $4.237+0.05$ & 1.8695 & 33.4486 \\
$25.792+0.10$ & $4.237-0.05$ & 1.8666 & 31.3885 \\
\hline \hline
\end{tabular}

samples. But the presence of microscopic roughness was verified by TEM measurements of replica films. The replicas were shadowed by Pt evaporated. The surface is revealed to be irregular, having a random texture with features separated by distances of the order of 20 to $60 \mathrm{~nm}$. This values correlated with our experimental data upon surface layer thicknesses. So the model of homogeneous manufactured layer for substrate microscopic roughness description works very well for our samples.

On the other side, we see that the traditional (BEMA) model, when the volume fractions in the surface layer were fixed to $50 \%\left(v_{a}=v_{s}=0.5\right)$, is not appropriate for our problem. The difference between experimental ( $\Psi_{\exp }$ and $\left.\Delta_{\text {exp }}\right)$ and calculated $\left(\Psi_{c}\right.$ and $\left.\Delta_{c}\right)$ data (Table 5) is more than the of the instrument accuracy. In the frame of this model the thickness of the surface roughness layer was determined to be $9 \mathrm{~nm}$. It is rather small value, which does not fit to real situation. The BEMA approximation with $v_{a}=0.11$ and $n_{f}=$ 1.869699 as parameters, which gives real $d_{f}$ value, may be an alternative to approximation by homogeneous manufactured layer. It is very difficult to detect so small fraction of air. Such kind of the model assumed the sample to consist of mixture GdGaG and voids. But TEM micrographs of backthinned samples did not confirmed the void presence in our samples.

\section{Conclusion}

In this study the refractive index $n_{f}$ and the layer thickness $d_{f}$ on each face of $\mathrm{GdGaG}$ plates have been determined by ellipsometry. The $n_{f}$ data was shown to be accurate to the third decimal point. Errors for $d_{f}$ are not more than $3 \%$. In comparison of two models, the model of homogeneous manufactured layer for substrate microscopic roughness description are found to represent adequately the experimental data, while BEMA approximation, previously used exclusively to model roughness in single-wavelength applications, gives poor results. These data may be used to characterize interface between ReFeG and $\mathrm{GdGaG}$ substrate.

\section{Acknowledgment}

This work is supported by International Science Foundation (Grant N QSU082009).

\section{References}

1. R.M.A.Azzam and N.M. Bashara, Ellipsometry and Polarized Light, North-Holland, Amsterdam, 1977, Chap.4, p.270.

2. D.A.G.Bruggeman, Ann.Phys.(Leip) 24 (1935) 636.

3. B.J.Gibbons,S.Trolier-McKinstry, IEEETrans.on Appl. Superconductivity 7 (1997) 2177.

4. Belyaeva A.I., Grebennik T.G., Proc.SPIE 3359 (1997) 401.

5. Belyaeva A.I.,Grebennik T.G.,Nastenko V.A.,Instr. and Exper. Techniques.40 (1997) 531 